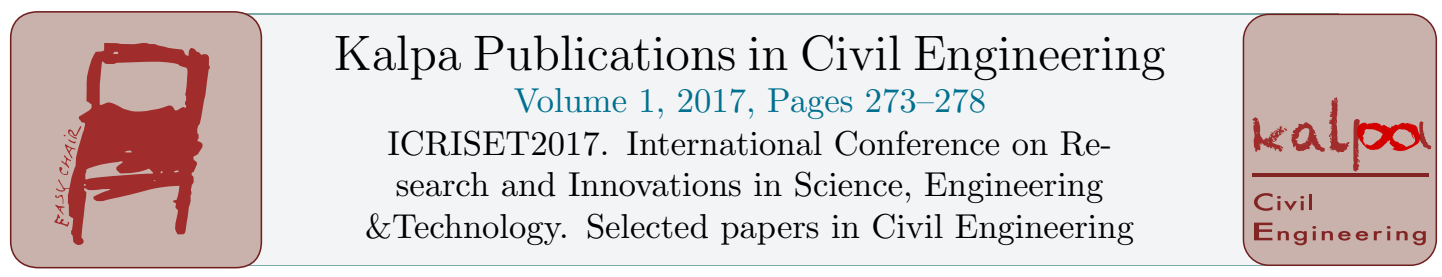

\title{
Study of Quadri Quintic Element Applicable to Deep Beams
}

\author{
Khevna B. Patel ${ }^{1}$, Atul N. Desai ${ }^{1}$ and Vishal A. Arekar ${ }^{1}$ \\ ${ }^{1}$ Department of Structural Engineering, B.V.M. Engineering college, Vallabh Vidyanagar, India \\ khevna6494 egmail.com, \\ andesai2bvmengineering.ac.in, vaarekar@bvmengineering.ac.in
}

\begin{abstract}
A lot of studies have been performed to explore the use and application of conforming elements in meshing of two and three-dimensional structures. Here, a combination of 5 nodes on $\mathrm{X}$ axis and 3 nodes on $\mathrm{Y}$ axis has been considered. Hence, the element used here is a 12 nodded rectangular nonconforming element, which can be termed as Quadri-Quintic nonconforming element. The development of a computer program to study the behavior of Quadri-Quintic nonconforming element and its application in meshing of deep beams has been targeted. The study may able to show that the proposed element gives results with higher accuracy and with faster convergence.
\end{abstract}

\section{Introduction}

Basically, the only method of structural analysis is the strain energy/ potential energy method. The applications of this method are developed as Slope deflection method, Moment distribution method, and Kani's method etc., basic concept of analysis being the same i.e. potential energy method. Finite element method is an extension of stiffness matrix method, which can be conveniently used for the analysis of two-dimensional and three-dimensional elements. The versatility of the finite element method has drawn much attention in the field of engineering. Numerous researches have been performed for the better and smarter applications of the method. As it involves lot of calculations, its growth is closely linked with the developments in computer technology. While solving a problem with FEM we come across repetitive work involving same formulae and calculations, as basic procedure remains same. In addition, working on large complex structure involves large matrices in Calculations. Keeping these points in mind a computer program has to be developed in the scope of the current work. Initially an excel spreadsheet is to be developed for simple meshing which can be extended for finer meshing using any programming language.

C.D. Modhera, G.J. Joshi, D. Soni, I.N. Patel, A.K. Verma, L.B. Zala, S.D. Dhiman, D.R. Bhatt, J.M. Rathod, B.C. Goradiya, M.S. Holia and D.K. Patel (eds.), ICRISET2017 (Kalpa Publications in Civil Engineering, vol. 1), pp. 273-278 


\section{Objectives of the study}

Basically, the concept of nonconforming element in the FEM is to be explored in this study. The behavior of Quadri-Quintic nonconforming element and its application in meshing of deep beams is to be studied. A computer program is to be developed using MS Excel for the same, which can be further extended for finer meshing using any programming language. A combination of Quadratic and Quintic i.e. 5 nodes on two sides and 3 nodes on other two sides are taken. This study may be able to obtain results with faster convergence.

\section{Literature review}

The plane quadrilateral element is a simple and widely used element in the analysis of plane problems and the design and development of low-order quadrilateral plane element with a high accuracy in coarse meshes has always been very popular in the Finite element method. Because of their flexibility and stability when compared with conforming finite element methods, nonconforming finite element methods have become very important and effective discretization methods for numerically solving, among others, high order elliptic problems, Stokes-like problems and ReissnerMindlin plate bending problems. Compared with nonconforming triangular finite elements, it is more difficult to construct nonconforming quadrilateral finite elements ${ }^{[3]}$. Huang and $\mathrm{Xie}^{[2]}$ analyzed a nonconforming 5 node transition element by finite element method in which the fifth node is placed at different positions as shown in figure 1.
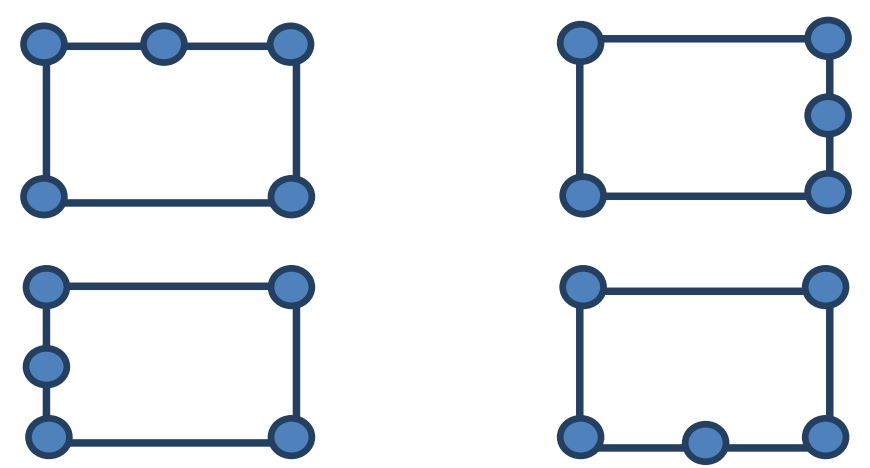

Figure 1: five nodded transition element

Douglas N. Arnold and Gerard Awanou ${ }^{[1]}$ gave a new, simple, dimension-independent definition of the serendipity finite element family. Hu Jun and Zhang Shang You ${ }^{[3]}$ proposed two families of nonconforming finite elements of any odd order and one family of nonconforming finite elements of any even order on quadrilateral meshes. Tinh Quoc Bui, Dam Quang Vo, Chuan zeng Zhang, Du Dinh Nguyen ${ }^{[7]}$ developed a consecutive interpolation quadrilateral element CQ4 and studied its applications in the stress analysis of 2D elastic structures. Juan Chen, Chong-Jun Li ${ }^{[5]}$ presented a paper in which a 24-DOF quadrilateral spline element for couple stress/strain gradient elasticity is proposed, which is a single displacement function. Tejpal Goda H., A. N. Desai, K. N. Sheth ${ }^{[6]}$ carried out a study of nonconforming element with six-nodes and compared it with the conforming four nodded rectangular element as shown in figure 2. Jaimin A. Joshi, A. N. Desai, K. N. Sheth ${ }^{[4]}$ carried $^{2}$ out a study of nonconforming quadrilateral element with 10 nodes as shown in figure 3 . 


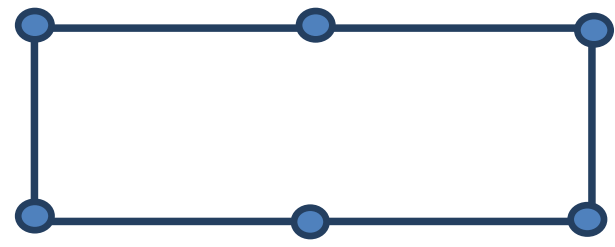

Figure 2: six nodded rectangular element

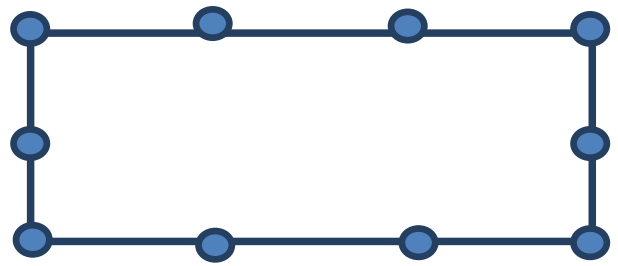

Figure 3: ten nodded rectangular element

\section{Quadri Quintic Nonconforming Element}

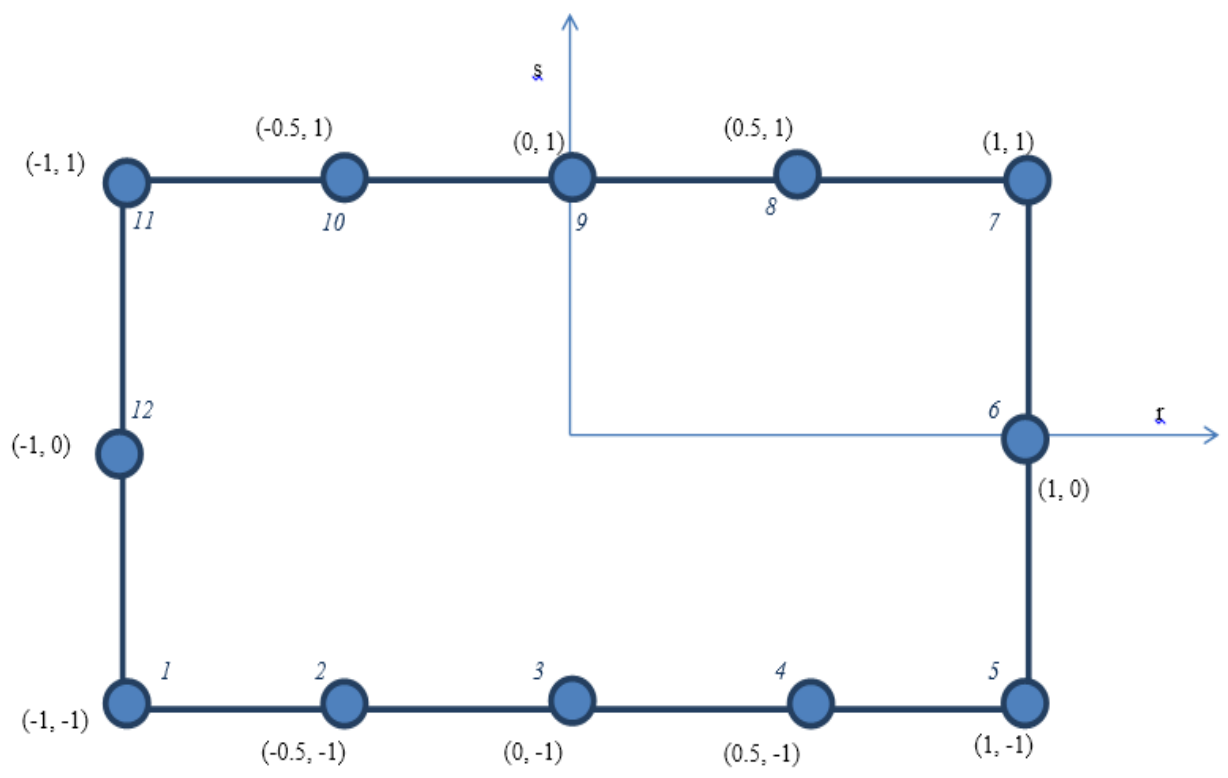

Figure 4: Quadri Quintic nonconforming element

Nonconforming elements are those, which have asymmetric distribution of nodes along both the axes. For example, adding three more nodes at equal intervals on the horizontal sides and one middle node on vertical sides of a basic four-nodded quadrilateral will make it a 12 nodded nonconforming rectangular element. Hence, adding and numbering 8 more nodes in anti-clockwise direction to make it a Quadri-Quintic nonconforming element as shown in figure 4, considering any one node, the value of its shape function at that particular node should be one and zeros at all other nodes. In addition, summation of all the shape functions should come to unity. Shape functions for this 12-nodded Quadri Quintic element can be derived as-

$$
\begin{aligned}
& \mathrm{N}_{2}=\frac{-4 r(1-s)\left(1-r^{2}\right)\left(\frac{1}{2}-r\right)}{3} \\
& \mathrm{~N}_{3}=2\left(1-r^{2}\right)(1-s)\left(\frac{1}{4}-r^{2}\right)
\end{aligned}
$$$$
\begin{aligned}
& \mathrm{N}_{4}=\frac{4 r(1-s)\left(1-r^{2}\right)\left(\frac{1}{2}+r\right)}{3} \\
& \mathrm{~N}_{6}=\frac{(1+r)\left(1-s^{2}\right)}{2}
\end{aligned}
$$ 


$$
\begin{array}{ll}
\mathrm{N}_{8}=\frac{4 r(1+s)\left(1-r^{2}\right)\left(\frac{1}{2}+r\right)}{3} & \mathrm{~N}_{10}=\frac{-4 r(1+s)\left(1-r^{2}\right)\left(\frac{1}{2}-r\right)}{3} \\
\mathrm{~N}_{9}=2\left(1-r^{2}\right)(1+s)\left(\frac{1}{4}-r^{2}\right) & \mathrm{N}_{12}=\frac{(1-r)\left(1-s^{2}\right)}{2}
\end{array}
$$

If $N_{1}^{\prime}, N_{2}^{\prime}, N_{3}^{\prime}$ and $N_{4}^{\prime}$ are shape functions for corner nodes of a standard four nodded quadrilateral element, shape functions for the corner nodes of a Quadri Quintic element can be revised as,

$$
\begin{array}{ll}
\mathrm{N}_{1}=\mathrm{N}_{1}^{\prime}-\frac{\mathrm{N}_{12}}{2}-\frac{3 \mathrm{~N}_{2}}{4}-\frac{\mathrm{N}_{3}}{2}-\frac{\mathrm{N}_{4}}{4} & \mathrm{~N}_{5}=\mathrm{N}_{5}^{\prime}-\frac{\mathrm{N}_{6}}{2}-\frac{3 \mathrm{~N}_{4}}{4}-\frac{\mathrm{N}_{3}}{2}-\frac{\mathrm{N}_{2}}{4} \\
\mathrm{~N}_{7}=\mathrm{N}_{7}^{\prime}-\frac{\mathrm{N}_{6}}{2}-\frac{3 \mathrm{~N}_{8}}{4}-\frac{\mathrm{N}_{9}}{2}-\frac{\mathrm{N}_{10}}{4} & \mathrm{~N}_{11}=\mathrm{N}_{11}^{\prime}-\frac{\mathrm{N}_{12}}{2}-\frac{3 \mathrm{~N}_{10}}{4}-\frac{\mathrm{N}_{9}}{2}-\frac{\mathrm{N}_{8}}{4}
\end{array}
$$

Now, suitability of this element to application to the deep beam analysis can be understood by considering a few points. Deep beams are subjected to non-linear stress distribution even in the elastic stage. It can be observed from the shape functions of the Quadri Quintic element that it can serve for the non-linear distribution. While deep beam behaves as a two dimensional member, the proposed nonconforming element is also a two dimensional element. First step in analysis is to find field variables. It is assumed that at any point inside the element, field variable is a function of field variables at nodal points of the element. The function that relates the field variables of internal points to the field variables at nodal points is called shape function or interpolating function. For twodimensional stress analysis the basic field variable is displacement.

$$
\mathrm{u}=\sum \mathrm{N}_{\mathrm{i}} \mathrm{u}_{\mathrm{i}} \quad \mathrm{v}=\sum \mathrm{N}_{\mathrm{i}} \mathrm{v}_{\mathrm{i}}
$$

Here ' $\mathrm{i}$ ' is the index number for nodes and $\mathrm{N}_{\mathrm{i}}$ is the shape function for $\mathrm{i}^{\text {th }}$ node. $\mathrm{u}$ and $\mathrm{v}$ denote the horizontal and vertical displacements respectively.

$\mathrm{u}=\sum \mathrm{N}_{\mathrm{i}} \mathrm{u}_{\mathrm{i}}=\mathrm{N}_{1} \mathrm{u}_{1}+\mathrm{N}_{2} \mathrm{u}_{2}+\mathrm{N}_{3} \mathrm{u}_{3}+\ldots+\mathrm{N}_{12} \mathrm{u}_{12}$

$\mathrm{v}=\sum \mathrm{N}_{\mathrm{i}} \mathrm{v}_{\mathrm{i}}=\mathrm{N}_{1} \mathrm{v}_{1}+\mathrm{N}_{2} \mathrm{v}_{2}+\mathrm{N}_{3} \mathrm{v}_{3}+\ldots+\mathrm{N}_{12} \mathrm{v}_{12}$

i.e. $\{\mathrm{u}\}=[\mathrm{N}]^{*}\{\delta\}$

Where,

$$
[N]=\left[\begin{array}{ccccccccc}
N_{1} & 0 & N_{2} & 0 & N_{3} & 0 & \ldots \ldots & N_{12} & 0 \\
0 & N_{1} & 0 & N_{2} & 0 & N_{3} & \ldots \ldots & 0 & N_{12}
\end{array}\right]
$$

And displacement,

$$
\{\mathrm{u}\}=[N] *\left\{\begin{array}{c}
\boldsymbol{u}_{1} \\
\boldsymbol{v}_{1} \\
\boldsymbol{u}_{2} \\
\boldsymbol{v}_{2} \\
\cdot \\
\cdot \\
\boldsymbol{u}_{12} \\
\boldsymbol{v}_{12}
\end{array}\right\}
$$

Now, the Jacobian matrix can be written as

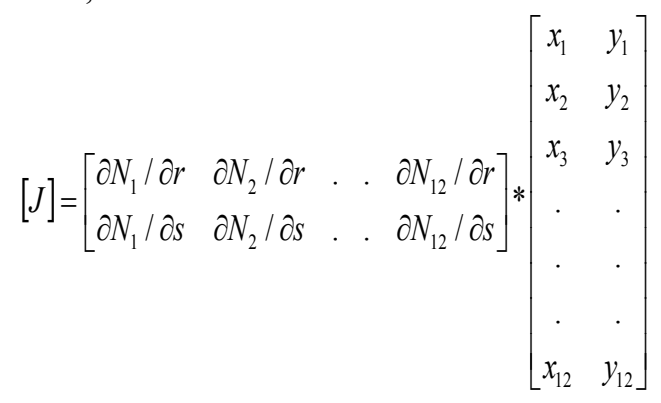


Here, $\left(\mathrm{x}_{\mathrm{i}}, \mathrm{y}_{\mathrm{i}}\right)$ are the geometric coordinates of the nodes. $\mathrm{r}$ and $\mathrm{s}$ are the isoparametric axes.

The Geometric matrix will be

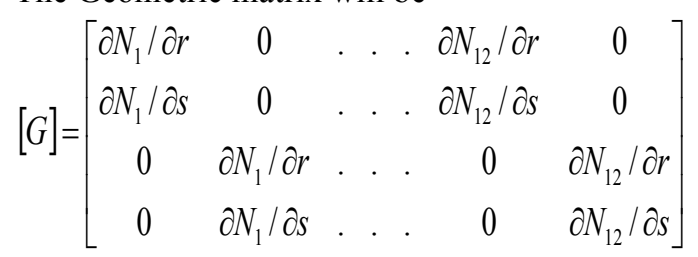

Strain-Displacement matrix is given by $[\mathrm{B}]=[\mathrm{A}][\mathrm{G}]$

Where

$$
[A]=\left[\begin{array}{cccc}
J_{11}^{*} & J_{12}^{*} & 0 & 0 \\
0 & 0 & J_{21}^{*} & J_{22}^{*} \\
J_{21}^{*} & J_{22}^{*} & J_{11}^{*} & J_{12}^{*}
\end{array}\right]
$$

The stiffness matrix is obtained by the following equation,

$[\mathrm{K}]=\mathrm{t} * \iint_{-1}^{1} B^{T} D B d A$

Where, $\mathrm{t}=$ width of the beam

$$
\begin{aligned}
& \mathrm{D}=3 * 3 \text { constitutive matrix } \\
& \mathrm{dA}=\mathrm{dx} \mathrm{dy} \\
& =|\mathrm{J}| * \mathrm{dr} * \mathrm{ds}
\end{aligned}
$$

In this study, the Gaussian quadrature approach of integration (three points) is used. This method has proved most useful in finite element work. The form of evaluating $I$ is shown below. Extension to two and three dimensions follows readily. Considering n point approximation,

$$
I=\int_{-1}^{1} f(\varphi) d \varphi=w_{1} f\left(\varphi_{1}\right)+w_{2} f\left(\varphi_{2}\right)+\cdots+w_{n} f\left(\varphi_{n}\right)
$$

Where $\mathrm{w}_{1}, \mathrm{w} 2 \ldots \mathrm{wn}$ are the weights and $\varphi_{1}, \varphi_{2}, \ldots, \varphi_{n}$ are the sampling points or Gauss points. Gaussian Quadrature of $n$ points will provide an exact answer if $f$ is the polynomial of order (2n-1). The Gauss points are located symmetrically with respect to origin and symmetrically placed points have the same weights. For a Quadri Quintic element, size of the geometric matrix G is $4 \times 24$ and the size of stiffness matrix is $24 \times 24$.

\section{Sample Meshing}

First sample meshing is done for a cantilever deep beam using a single element. The beam is $1 \mathrm{~m}$ long, $0.5 \mathrm{~m}$ deep and $0.15 \mathrm{~m}$ wide. The beam is subjected to a point load at the free end. The procedure of finite element analysis is performed by the program developed in MS Excel. Three point Gauss Quadrature approach is used for the integration. Values of deflections are obtained at each node and compared with the standard deflection value for the cantilever under point load. i.e.

$$
\delta=\frac{P L^{3}}{3 E I}
$$




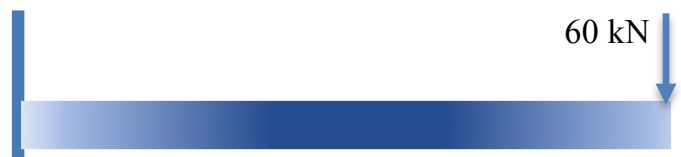

Figure 4: sample beam

\begin{tabular}{|l|l|l|}
\hline & MS-EXCEL & FORMULA \\
\hline DEFLECTION & $0.525 \mathrm{~mm}$ & $0.467 \mathrm{~mm}$ \\
\hline
\end{tabular}

Table 1: results

The percentage error occurred here is $12.3 \%$. The difference in the results by both methods can be explained by the fact that the standard deflection formula does not count for shear deformations whereas present study has considered the shear deformations.

\section{Conclusion}

It is concluded that the proposed Quadri Quintic Element is stable and applicable to analysis of deep beams. Higher degree of accuracy may be obtained with finer meshing.

\section{References}

1) Douglas N. Arnold and Gerard Awanou, "The Serendipity Family of Finite Elements", Foundations of computational mathematics, March 2011

2) F. Huang and X. Xie, "A modified nonconforming 5-node quadrilateral transition finite element". Advances in Applied Mathematics and Mechanics, December 2010

3) Hu Jun and Zhang Shang You, "Nonconforming finite element methods on quadrilateral meshes", Science China Mathematics, December 2013

4) Jaimin A. Joshi, A. N. Desai, K. N. Sheth, "Study of quadri-cubic element applicable to deep beam", National Conference on Emerging Vistas of Technology (NCEVT-'15)

5) Juan Chen and Chong-Jun $\mathrm{Li}$, “A quadrilateral spline element for couple stress/strain gradient elasticity”, Computers and Structures, April 2014

6) Tejpal Goda H., A. N. Desai, K. N. Sheth, "Study of quadri-linear (nonconforming) element", National Conference on Recent Advances in Civil and Structural Engineering (RACSE-'14)

7) Tinh Quoc Bui, Dam Quang Vo et al., "A consecutive-interpolation quadrilateral element (CQ4): Formulation and applications “, Finite Elements in Analysis and Design , March 2014 